\title{
Statistics of auroral Langmuir waves
}

\author{
M. Samara ${ }^{1, *}$, J. LaBelle ${ }^{1}$, and I. H. Cairns ${ }^{2}$ \\ ${ }^{1}$ Department of Physics and Astronomy, Dartmouth College, Hanover, NH, USA \\ ${ }^{2}$ School of Physics, University of Sydney, NSW 2006, Australia \\ *now at: Space Science and Engineering Division, Southwest Research Institute, San Antonio, TX, USA
}

Received: 6 July 2007 - Revised: 5 August 2008 - Accepted: 22 October 2008 - Published: 3 December 2008

\begin{abstract}
The Physics of Auroral Zone Electrons II (PHAZE II) sounding rocket was launched in February 1997 into active pre-midnight aurora. The resulting high frequency wave data are dominated by Langmuir waves. Consistent with many previous observations the Langmuir waves are sporadic, occurring in bursts lasting up to a few hundred ms. We compute statistics of the electric field amplitudes of these Langmuir waves, with two results. First, the shape of the distribution of running averages of the electric field amplitudes remains approximately stationary for a large range of widths of running average less than $\sim 0.3 \mathrm{~ms}$ and for a large range of widths exceeding about $1 \mathrm{~ms}$. The interpretation of this transition timescale is unclear but appears unlikely to be of instrumental origin. Second, for 2.6-ms running averages, corresponding to the latter range, the distribution of the logarithm of electric field amplitudes matches a Gaussian form very well for all nine cases studied in detail, hence the statistics are lognormal. These distributions are consistent with stochastic growth theory (SGT).
\end{abstract}

Keywords. Ionosphere (Auroral ionosphere; Electric fields and currents; Wave propagation)

\section{Introduction}

Auroral electron beams generate Langmuir waves over a wide range of altitudes, from the bottomside of the F-region to thousands of $\mathrm{km}$. At the lower end of this range rocketborne wave receiver data reveal that these waves occur in bursts lasting from $\approx 1 \mathrm{~ms}$ to a few hundred ms (e.g. Boehm, 1987; Ergun et al., 1991a,b; McAdams et al., 1999). The frequency structure of the waves depends on the ratio of the electron plasma frequency to the electron cyclotron frequency $\left(f_{p e} / f_{c e}\right)$ (e.g. Beghin et al., 1989; McAdams et al.,

Correspondence to: M. Samara

(msamara@swri.edu)
1999; McAdams and LaBelle, 1999). For $f_{p e}<f_{c e}$, banded structures occur at frequencies below $f_{p e}$, with bands terminating in intense wave bursts at $f=f_{p e}$. McAdams et al. (1999) proposed that these bands result from conversion of Langmuir waves into whistler waves as they move on a single dispersion surface with constant frequency through an inhomogeneous plasma: the banded structure results from the causative Langmuir waves being sporadic rather than continuous. The banded structure therefore presents further evidence that the Langmuir waves occur in localized packets. Beghin et al. (1989) assert a similar interpretation for waves observed below $f_{p e}$ in underdense conditions encountered by the Aureole-3 satellite.

Many theory and simulation papers address the bursty nature of Langmuir waves in the auroral ionosphere. Ergun et al. (1991b) interpret bursty Langmuir wave data in terms of modulational instabilities involving lower hybrid or ion Bernstein waves. Newman et al. (1994a) considered linear aspects of the wave excitation and dispersion, both in the overdense and underdense cases. Newman et al. (1994b) developed a nonlinear turbulence theory for the resulting Langmuir waves based on modified Zakharov equations that predict aspects of the observed bursty and modulated wave fields. Muschietti et al. (1995) investigated the kinetic interaction of Langmuir wave packets with streaming energetic electrons, using low-noise self-consistent particle-incell (PIC) simulations with open boundary conditions and weak field amplitudes. They showed that the interaction can lead to wave localization, and applied the results to wave observations in the auroral zone. Kinetic localization of beam-driven Langmuir waves was also extended to parameters relevant to the electron foreshock using PIC simulations with relaxed conditions (i.e. allowing for ion dynamics) (Muschietti et al., 1996). Most recently, interest in beam-driven wave turbulence at intermediate altitudes in the aurora, above $2000 \mathrm{~km}$, has focussed on electron hole formation, directly observed with FAST satellite instruments

Published by Copernicus Publications on behalf of the European Geosciences Union. 
(Ergun et al., 1998), extensively simulated (Goldman et al., 1999; Newman et al., 2001; Oppenheim et al., 1999, 2001), and modeled analytically by the BGK model (Muschietti et al., 1999a,b, 2000, 2002).

In this paper, we investigate the statistics of the amplitudes of Langmuir wave bursts observed in the ionosphere under underdense conditions $f_{p e}<f_{c e}$ using a rocket-borne wave receiver. The inspiration for this work comes from investigations of Langmuir waves in the electron foreshock, where they are generated by electrons streaming away from the bow shock that develop a beam distribution function by time-of-flight effects. In this region nonlinear processes such as modulational instabilities and strong turbulence wave collapse (for example, Zakharov, 1972; Nicholson et al., 1978; Robinson, 1997) and nonlinear three-wave decay into daughter Langmuir and ion acoustic waves (Cairns and Melrose, 1985; Lin et al., 1986) do not appear to saturate the beam instability because the observed electric fields and predicted beam speeds are typically too low (Cairns and Robinson, 1992; Cairns et al., 1998). From statistics of the Langmuir wave amplitudes measured with the ISEE-1 satellite wave receiver, Cairns and Robinson $(1997,1999)$ find that the wave fields follow a log-normal distribution. Such a distribution is predicted by Stochastic Growth Theory (SGT), developed for the bursty Langmuir waves driven by an electron beam in the source region of solar type III radio bursts (Robinson, 1992; Robinson et al., 1993). The statistical analyses of Langmuir waves in type III source regions and Earth's foreshock (Robinson et al., 1993; Cairns and Robinson, 1997, 1999) suggest that SGT is the appropriate theoretical framework for understanding Langmuir turbulence in these two regions.

The auroral plasma is much more strongly magnetized than either the solar wind or the electron foreshock, but it represents another natural environment in which an electron beam penetrates a background plasma and generates Langmuir waves. Statistical analyses such as those employed by Robinson et al. (1993) and Cairns and Robinson (1997, 1999) may provide a valuable constraint on theories developed to explain auroral Langmuir turbulence. Indeed, Cairns and Menietti (2001) and Burinskaya et al. (2000) analyse the statistics of Langmuir-like waves driven by electron beams in the more strongly magnetized plasma of the polar cap, finding agreement with SGT. On the other hand, nonlinear three-wave processes involving Langmuir and lower hybrid waves arguably cause modulation of auroral Langmuir waves observed in satellite and rocket experiments (Ergun et al., 1991b; Bonnell et al., 1997; Khotyaintsev et al., 2001; Lizunov et al., 2001).

In this paper we undertake a statistical analysis of the wave fields of a selected set of Langmuir wave bursts detected at $600-700 \mathrm{~km}$ altitude in the auroral region using instruments on board the PHAZE II sounding rocket. Section 2 describes the instrumentation used to collect the data; Sect. 3 presents the statistical analysis and resulting fitted parameters; and
Sect. 4 is a discussion of the results in terms of possible theoretical frameworks for understanding auroral Langmuir turbulence.

\section{Instrumentation}

The PHAZE II sounding rocket was launched to an apogee of $945 \mathrm{~km}$ in active pre-midnight aurora on 10 February 1997 , at 05:17 UT. The spin axis of the payload was aligned parallel to the background magnetic field and the spin rate was $0.6 \mathrm{~Hz}$. It carried a full complement of particle and field instruments, provided by UNH and Cornell University, respectively (Arnoldy et al., 1999). Dartmouth College provided a High Frequency Electric Field Instrument (HFE). These high frequency field measurements provided information about the electron density through observations of cutoffs and resonances. However, the high frequency wave fields are also intrinsically interesting because in the auroral ionosphere they play a significant role in beam-plasma interactions.

The sensor for the HFE consisted of two $5 \mathrm{~cm}$ cylindrical probes mounted $30 \mathrm{~cm}$ apart on an approximately 1-m fixed axial boom projecting forward from the nose of the rocket payload, parallel to the rocket spin axis and hence approximately parallel to the background magnetic field. The antenna was therefore on the same field line as the payload. For reference, in the topside ionosphere the electron gyroradius is of order a few centimeters, the ion gyroradius of order a few meters, and the Debye length typically the order of a centimeter but was closer to ten centimeters for the low electron densities encountered by SIERRA (McAdams and LaBelle, 1999).

The HFE continuously measured the waveform of the electric field with $5 \mathrm{MHz}$ bandwidth, which was transmitted to the ground station using a wide band analog telemetry (TM) transmitter; for details, see McAdams et al. (1999). This measurement strategy required using an automatic gain control (AGC) because of the limited dynamic range of the wideband telemetry link. The AGC varies the amplification of the HF signals during the flight in order to assure that the transmitter is always fully modulated and therefore makes optimal use of the telemetry, and as a result the variable AGC gain must be folded in to recover the waveform and spectral signatures. Previous flights observed whistler mode signals below $400 \mathrm{kHz}$ which can force the AGC into a low gain state and make the receiver insensitive to weaker HF signals of interest at higher frequencies. Therefore, a 5-pole high pass filter with $3-\mathrm{db}$ point at $400 \mathrm{kHz}$ was used to ensure that the auroral hiss and other low frequency noise would not set the gain level of the AGC. In addition, a low pass filter at $5 \mathrm{MHz}$ was included in the signal path to avoid interference with other TM links and also to avoid aliasing when the signal was digitized.

The AGC was implemented with analog electronics which detected the peak level of electric field fluctuations; when 


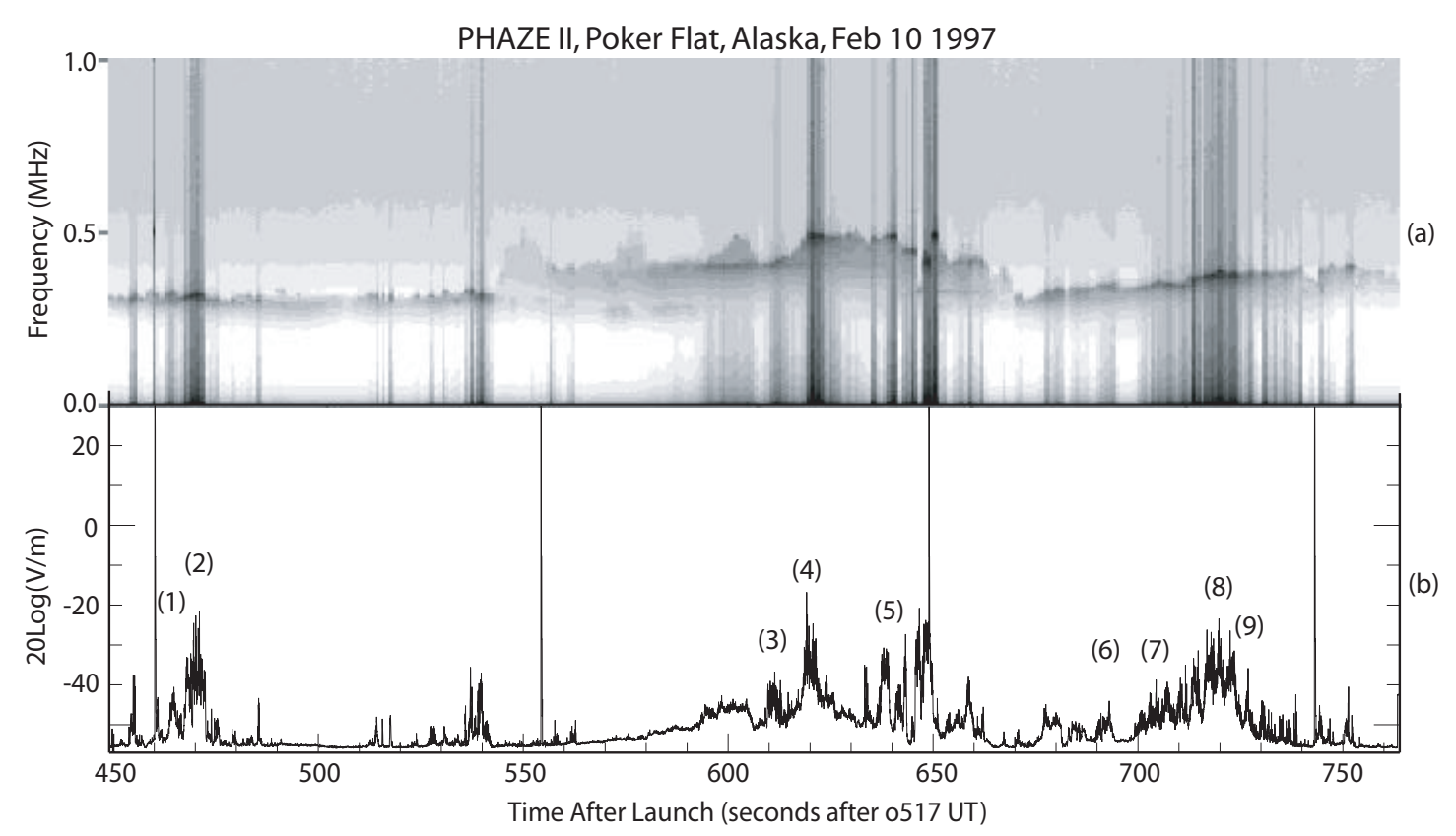

Fig. 1. (a) Sequence of Fast Fourier Transform (FFT) spectra of the measured HF electric field with frequency resolution of $9.76 \mathrm{kHz}$. There is a $70 \mathrm{~dB}$ difference between white and black on the grayscale. The horizontal axis is time since launch in seconds, and the vertical axis is frequency in MHz. Vertical bands, such as at $640 \mathrm{~s}$ or $670 \mathrm{~s}$, arise from the action of the AGC as discussed in the text. (b) The logarithm of the absolute value of the amplitude of the electric field for the same 449-763 s time period. The data are expressed in decibel units $\left(20 \log _{10}(\mathrm{~V} / \mathrm{m})\right)$. These data represent $6 \mathrm{~ms}$ averages and only one out of four points is displayed. The AGC has been accounted for in both panels; calibration signals are removed from the top panel.

the peak level exceeded a threshold, the circuit responded by reducing the gain of a logarithmic amplifier. The peak detector had a time constant of $1.6 \mathrm{~ms}$; i.e. when it exceeded the threshold, the peak electric field signal was passed through an RC low-pass filter with a time constant $\tau=1 / R C=1.6 \mathrm{~ms}$, resulting in a slowly varying voltage which controlled the $\log$ amplifier gain. As a result, increases or decreases of the electric field with time scale less than $1.6 \mathrm{~ms}$ were not effectively regulated by the AGC but were registered directly in the raw HF signal level, while variations of the signal with time scale $1.6 \mathrm{~ms}$ or longer were effectively regulated by the AGC. The quasi-DC control voltage was independently sampled at a $2 \mathrm{kHz}$ rate, sufficient to allow accurate reconstruction of the waveform. Significant distortion could occur only if an unregulated impulsive increase in the signal exceeded the range of the analog to digital converter, or if an unregulated rapid decrease in the signal was so severe that the signal level fell below the noise level of the system. The statistics of the raw HF signals, discussed in detail below, showed that occurrences of such distortion were rare during the events studied for this paper and had a negligible effect on the analysis of signal levels presented below.

A variety of wave modes was observed during the PHAZE II flight, including whistler mode signals below $f_{p e}$ and harmonics of them near $1.1 \mathrm{MHz}$ (LaBelle et al., 1999), band and chirp phenomena near the plasma frequency
(McAdams et al., 1999, 2000; McAdams and LaBelle, 1999), diffuse signals near $f_{p e}$ near the F-peak and man-made interference signals above $f_{p e}$. In this paper we focus on the $\mathrm{HF}$ waves between $200 \mathrm{kHz}$ and $500 \mathrm{kHz}$ above $\approx 780 \mathrm{~km}$ altitude, which are dominated by Langmuir waves at or near $f_{p e}$. Due to unusually low densities during this flight, $f_{p e}$ ranged from $200-500 \mathrm{kHz}$.

\section{Data analysis}

Figure 1 is an overview of the active part of the flight, from 449 to $763 \mathrm{~s}$ after launch. Apogee is at $556 \mathrm{~s}$ and $945.3 \mathrm{~km}$. During this period the rocket penetrated multiple auroral arcs as indicated by inverted $\mathrm{V}$ signatures in $1-10 \mathrm{keV}$ electron spectra (Arnoldy et al., 1999; Lynch et al., 1999). Figure 1a (top panel) represents a sequence of Fast Fourier Transform (FFT) spectra of the measured HF electric field. There are 512 points per FFT, skipping 1280000 points between each transform, and the digitization rate is $5 \mathrm{MHz}$. These numbers translate to a time resolution of one spectrum per $0.256 \mathrm{~s}$ and a frequency resolution of $9.76 \mathrm{kHz}$. The intensity of the grayscale corresponds to the power spectral density of the FFT, with a difference of $70 \mathrm{~dB}$ between white and black. The horizontal axis is time since launch in seconds, and the vertical axis is frequency in MHz. 
The signals shown in Fig. 1 have been adjusted to account for the variable AGC level and associated amplification of the instrument. This a process that entails multiplying by a large number when the gain is low and a small number when the gain is high. When strong natural signals near $f_{p e}$ cause the instrument to reduce the gain, the result is a reduced signal to noise ratio on the telemetry link for the weak signals away from $f_{p e}$. Then, adjusting the signal strength for the gain leads to the telemetry noise appearing as a vertical bar of high power spectral density. Examples are near 620 and $640 \mathrm{~s}$.

In Fig. 1a calibration signals, which are used for correct timing and amplitude calibration, have been removed; that is, any spectra including such signals are not displayed. Since the duty cycle of these calibrations is of order $1 \%$, removing them has negligible effect on the plot.

The upper frequency cutoff of the waves near 250$500 \mathrm{kHz}$ is interpreted as the local plasma frequency $f_{p e}$ (McAdams et al., 1998). This active part of the flight corresponds to underdense plasma $\left(f_{p e}<f_{c e}\right)$, for which $f_{p e}$ appears as a lower cutoff of LO-mode signals. For a nominal value of $f_{p e}=400 \mathrm{kHz}$ the plasma density is approximately $2000 \mathrm{~cm}^{-3}$. In addition to the cutoff effect, Langmuir waves near $f_{p e}$ are occasionally excited to large amplitudes, for example near time $470 \mathrm{~s}$. The bursty nature of these Langmuir waves is evident in the spectrogram and even more obvious in the time series data (bottom panel).

Figure $1 \mathrm{~b}$ (bottom panel) shows the logarithm of the $a b-$ solute value of the amplitude of the electric field for the same 449-763 s time period. These data represent $6 \mathrm{~ms}$ averages decimated by a factor of four. The AGC has been accounted for and the data are expressed in decibel units, given by $20 \log _{10}(E)$ where $E$ is the electric field, in $\mathrm{V} / \mathrm{m}$, obtained from the measured antenna voltage and the effective antenna length is assumed to equal the physical length of $30 \mathrm{~cm}$. Peaks at 460, 555, 650 and $745 \mathrm{~s}$ are artificially injected calibration signals. The time series shows that a large range of amplitudes occurs: the rms level varies over more than an order of magnitude, typically being in the range $3-32 \mathrm{mV} / \mathrm{m}$, and the peak amplitude ranges up to approximately $158 \mathrm{mV} / \mathrm{m}$. Assuming $n=2200 \mathrm{~cm}^{-3}$, inferred from the observed $f_{p e}$, and $T_{e}=3000 \mathrm{~K}$, a typical auroral F-region value, this observed range of electric field amplitudes corresponds to $\epsilon_{0} E^{2} / n k_{B} T_{e} \ll 1$, in the range $0.9 \times 10^{-6}-2.5 \times 10^{-3}$.

As expected from previous observations cited above, the Langmuir waves occur in bursts. For careful study, we selected nine such bursts that were well defined, relatively large amplitude and had sufficiently long duration to allow good statistics. This last characteristic was accomplished by requiring at least 270 independent 6 -ms electric field measurements. These bursts are denoted 1 through 9 in Fig. 1b.

Figure $2 \mathrm{~b}$ (bottom panel) shows the electric field amplitude, corrected for the AGC, versus time for "event 1 " in Fig. $1 \mathrm{~b}$, corresponding to time $465 \mathrm{~s}$ and altitude $915 \mathrm{~km}$. These data have been digitally filtered using a band pass filter with a lower end of $100 \mathrm{kHz}$ and an upper end of $2.5 \mathrm{MHz}$, in addition to the on-board analog filters at $100 \mathrm{kHz}$ and $5 \mathrm{MHz}$. Throughout the sample period the dominant signal is the Langmuir wave at about $400 \mathrm{kHz}$, well within the pass band. The data shown are decimated by a factor of 100 , and despite sub-sampling, the bursty nature of the waves is evident.

To obtain statistical distributions of electric fields analogous to the analysis of Cairns and Robinson (1997), one can use either straight averages or running averages of the measured electric field amplitudes. Both averaging methods are standard methods of smoothing or filtering time series. There is effectively no difference between them, but we elected to calculate running averages. In both forms of averaging longer averaging times imply a better estimate of the electric field if it is stationary over the averaging time, as well as effectively filtering out variations on time scales faster than the averaging time. However, when statistics of the averaged quantities are presented, one must evaluate statistical uncertainty somewhat differently in the two cases. We treat each sample or average of distinct samples as an independent measurement and estimate the random statistical uncertainty as $\sqrt{N_{\text {eff }}} / N_{\text {eff }}$, where $N_{\text {eff }}$ is the effective number of independent measurements. Thus, because samples are in common between successive running averages, we consider the effective number of measurements to be reduced in proportion to the length of the running average, and the uncertainty is correspondingly larger. Specifically,

$N_{\text {eff }}=N_{\text {samp }} \Delta t / T$,

where $N_{\text {samp }}$ is the total number of samples, $\Delta t$ the time separation between samples, and $T$ the averaging time. In subsequent analyses, we use this method to estimate the statistical uncertainties.

The top panel of Fig. 2 displays the same data as the bottom panel, but in histogram form: the horizontal axis represents values of electric field amplitude into which data have been binned, and the vertical axis gives the number of occurrences in each bin. The amplitude axis of the histogram is linear in electric field. Ten curves are shown, corresponding to widths of running averages ranging from $1.2 \mu \mathrm{s}$ to $26 \mathrm{~ms}$. For larger running averages, although not obvious in Figs. 2 and 3 , the average electric field converges to values of $4.1 \mathrm{mV} / \mathrm{m}$ and $15.6 \mathrm{mV} / \mathrm{m}$, respectively. A long tail in the distribution beyond the right end of the figure implies that the average value is larger than it appears, especially for short running average time scales.

The different running averages suggest two different regimes based on the time scale of the running average. For time scales between 0.0012 and $0.026 \mathrm{~ms}$, the histograms are bunched together, from 0.12 to $0.26 \mathrm{~ms}$ they make a rapid transition, and then from 1.2 to $26 \mathrm{~ms}$ they are again similar. The transition lies near $0.3 \mathrm{~ms}$. We have done this analysis for all nine events with similar results. For instance, Fig. 3 shows the results for event 2 , which indicates a similar transition. For reference, the instrumental AGC time constant is 

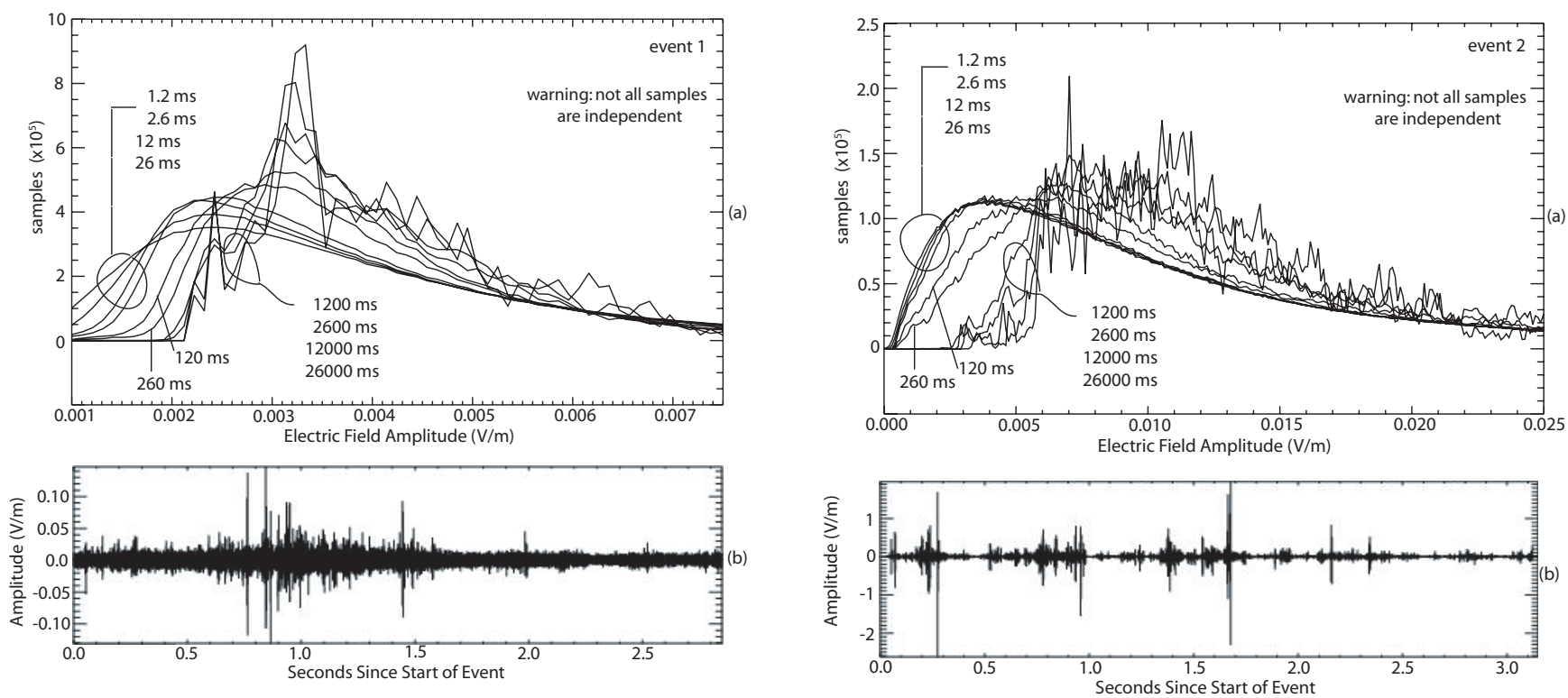

Fig. 2. (a) Electric fields of the the Langmuir burst denoted as event 1 in Fig. 1b, in histogram form: the horizontal axis represents values of the electric field amplitude into which data have been binned, and the vertical axis gives the number of occurrences in each bin. Ten curves are shown, corresponding to widths of running averages of electric field amplitudes ranging from $1.2 \mu \mathrm{s}$ to $26 \mathrm{~ms}$ (from top to bottom). (b) Time series of the sampled electric field amplitudes in event 2, corrected for the automatic gain control and decimated by a factor of 100 .

$1.6 \mathrm{~ms}$, about five times this transition timescale. The origin of this unexpected transition feature is unknown, but several possibilities, including possible instrumental effects, are discussed in Sect. 4 below.

Figure 4 presents an expanded and modified view of the histogram for event 2 , with a running average period of $2.6 \mathrm{~ms}$ that exceeds both the transition time $(0.3 \mathrm{~ms})$ described above and the AGC time constant $(1.6 \mathrm{~ms})$. The $\mathrm{x}-$ axis is the logarithm of the electric field amplitude while the $y$-axis is the logarithm of the effective number of occurrences, $N_{\text {eff }}$, defined in (1) above. The error bars give the statistical uncertainty $\sqrt{N_{\text {eff }}}$ predicted for counting statistics that take into account the number of independent measurements, also as described above.

In Fig. 4 and subsequent analyses, we account for the AGC and pass the data through a band pass digital filter with pass band $0.1-2.5 \mathrm{MHz}$. We conducted this analysis twice; once digitally adjusting for the instrumental high pass filter at $400 \mathrm{kHz}$ and once without the adjustment. We observe no difference between these two analyses, even though event 1 , in which the Langmuir waves occur well below $400 \mathrm{kHz}$, should suffer maximally from any effect of the instrumental filter. We also checked the other eight events with the same result. Hence in Figs. 4 and 5 we have not adjusted the waveform for the instrumental high pass filter.

Fig. 3. Time series and histogram of running-averaged samples for the Langmuir burst denoted as event 2 in Fig. 1b, in the same format as Fig. 2.

The measured electric fields appear broadly peaked near $10 \mathrm{mV} / \mathrm{m}(\log E=-2.0)$. Inspired by work done on statistics of Langmuir waves in the foreshock (Cairns and Robinson, 1997, 1999), we used the weighted least-squares method to fit the data over the entire observed range (typically between $\approx 3$ and $100 \mathrm{mV} / \mathrm{m}(\log E=-2.5$ to -1.0$)$ to a Gaussian form:

$y=a_{0} e^{-\left(x-a_{1}\right)^{2} / 2 a_{2}^{2}}$.

Here $x=\log E$. A good fit to a Gaussian would imply a $\log$ normal electric field distribution. The resulting fit is shown in Fig. 4 as a light line. The fitted parameters are given in the first three columns of Table 1 along with reduced $\chi^{2}$ values which provide a relative estimate of goodness-of-fit. The reduced $\chi^{2}$ values, calculated for the entire observed range of fields, are less than or of order unity in all cases, implying that that the lognormal provides a very good fit with high statistical significance.

Figure 5 shows histograms with error bars for all nine events. As in Fig. 4, each histogram corresponds to a $2.6 \mathrm{~ms}$ running average, and light lines indicate the best-fit Gaussian over the range of observed electric fields. The fit parameters are given in Table 1, including reduced $\chi^{2}$ values over the fitted range of fields. The low normalized $\chi^{2}$ values, all but one smaller than unity, suggest that our estimated correction to the uncertainty, shown as error bars in Figs. 4 and 5, may be actually somewhat overestimating the statistical uncertainty.

In some cases in Fig. 5, most noticeably events 3 and 7, the histogram data appear to depart from the lognormal fit at small $E$, with fewer samples observed than predicted by the fit. These departures occur for electric field amplitudes 
Table 1. Fit parameters for all nine events, as described in the text.

\begin{tabular}{cccccc}
\hline event & time (s) & $a_{0}$ & $a_{1}$ & $a_{2}$ & red. $\chi^{2}$ \\
\hline one & $463.55-466.4$ & 1.54 & -2.42 & 0.225 & 0.38 \\
two & $469.25-472.4$ & 1.31 & -1.95 & 0.387 & 0.32 \\
three & $609.35-612.95$ & 1.56 & -2.27 & 0.280 & 0.47 \\
four & $618.41-621.44$ & 1.42 & -1.82 & 0.284 & 0.16 \\
five & $637.1-639.05$ & 1.27 & -1.85 & -0.237 & 0.14 \\
six & $689.9-694.07$ & 1.84 & -2.53 & 0.182 & 0.58 \\
seven & $700.4-713.0$ & 2.00 & -2.39 & 0.291 & 1.18 \\
eight & $716.0-725.0$ & 1.79 & -1.97 & 0.420 & 0.48 \\
nine & $726.02-728.18$ & 1.42 & -2.41 & 0.205 & 0.42 \\
\hline
\end{tabular}

very near the instrumental noise level, where the background noise, rather than Langmuir waves may determine the shape of the histogram. In other cases in Fig. 5, most noticeably events 8 and 9, an additional peaked feature appears at lower fields than the main peak, which obviously cannot be predicted by a simple lognormal form. These features slightly exceed the $1-\sigma$ uncertainty estimate but are well within $2-\sigma$ and do not cause the reduced $\chi^{2}$ to significantly exceed 1 . Accordingly, the significance of these departures is not sufficient, based on these data, to warrant attention.

In summary, two features emerge from statistical analysis of nine intervals of auroral Langmuir wave bursts observed with the HF waveform receiver on PHAZE-II. First, analysis of the probability distribution of the Langmuir wave electric fields suggests a characteristic time scale associated with the wave bursts: for more than an order of magnitude in width of running average, $1-26 \mu \mathrm{s}$, the distributions resemble each other before making a rapid transition to a different form, which then governs the distribution for more than an order of magnitude in width of running average, $1-26 \mathrm{~ms}$. This seems to imply a transition timescale of $\tau \sim 0.3 \mathrm{~ms}$, which far exceeds filter time constants and is about a factor of five below the AGC time constant of $1.6 \mathrm{~ms}$.

The second result concerns the statistics of the amplitudes of the Langmuir waves for running averages exceeding the transition time, determined from studying distributions made using a $2.6 \mathrm{~ms}$ running average. In all nine distributions studied, a lognormal form matches the observed distribution very well and with high statistical significance across the range of observed fields exceeding the instrumental noise level.

\section{Interpretation}

The PHAZE-II measurements confirm previous observations showing the bursty nature of auroral Langmuir waves, with wave bursts occurring on time scales of 1 to $100 \mathrm{~ms}$. These Langmuir wave amplitude variations may be temporal or spatial; neither possibility can be excluded based on the data from a single-point sounding rocket platform, although consideration of the wave dispersion relation provides some

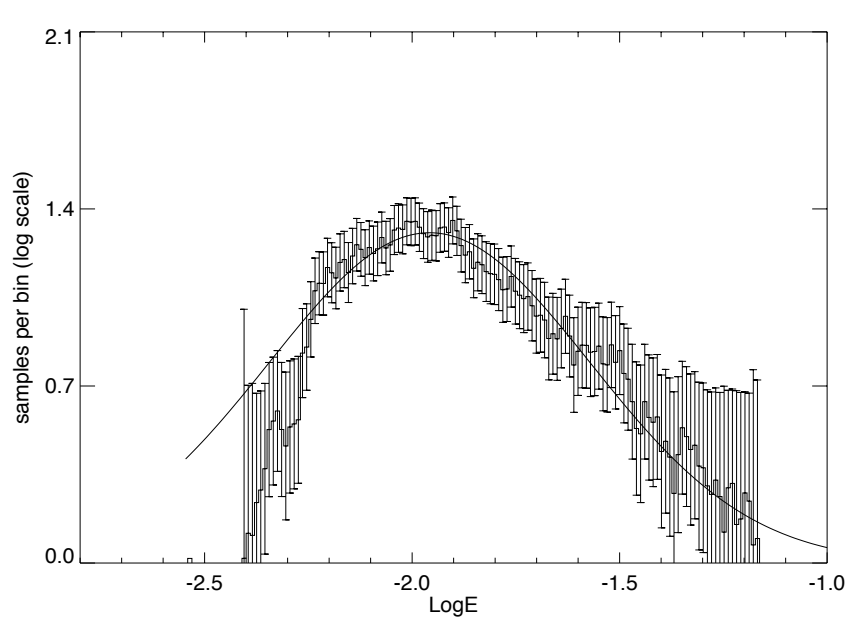

Fig. 4. Histogram for event 2 corresponding to $2.6 \mathrm{~ms}$ width for the running average. The $\mathrm{x}$-axis is the logarithm of the electric field amplitude while the $y$-axis is the logarithm of the number of occurrences. Error bars indicate the statistical uncertainty based on counting statistics, as explained in the text.

hints. If the waves are generated by auroral electrons with energies up to a few $\mathrm{keV}$ observed with electron detectors on the same rocket (Arnoldy et al., 1999), then the resonance condition for $\sim 0.5-1 \mathrm{MHz}$ plasma waves implies parallel wavelengths of tens of meters. Taking $k_{\|} / k_{\perp} \gg 1$ for waves within a few percent of $f_{p e}$, the perpendicular wavelength must be hundreds of meters, whereas interpretation of the $\sim 1-100 \mathrm{~ms}$ wave packets as spatial would imply perpendicular scales of $<100 \mathrm{~m}$ for the perpendicular rocket speed of $\sim 1 \mathrm{~km} / \mathrm{s}$. However, the parallel dynamics provide no such constraint on the interpretation: the Langmuir group velocity parallel to the magnetic field, $v_{g \|}=\partial \omega / \partial k_{\|}=3 \omega_{p e} k_{\|} \lambda_{d}^{2}$ is of order $5-25 \mathrm{~km} / \mathrm{s}$ for the parameters of the observed waves, much greater than the parallel rocket velocity. Hence, a $100 \mathrm{~ms}$ wave packet, if spatial, would correspond to scales up to kilometer scales, larger than the parallel wavelength by an ample margin. Unfortunately, no direct measurements using wave interferometry are available to test the hypothesis that the Langmuir wave bursts are temporal rather than spatial.

Several plausible reasons exist for the change in the form of the time-averaged field statistics near an averaging time of $0.3 \mathrm{~ms}$. Possible instrumental causes include the various analog and digital filters, which have time constants of order $1 / 400 \mathrm{kHz}, 1 / 100 \mathrm{kHz}$, and $1 / 2.5 \mathrm{MHz}$, far shorter than the transition timescale. Another possible instrumental effect is the AGC, which has a time constant of $1.6 \mathrm{~ms}$, about a factor of five higher than the transition timescale. The AGC could only produce artificial distortions of the measured waveforms if sufficiently rapid large variations in the envelope of the Langmuir wave occur. A large enough increase in the envelope within $1.6 \mathrm{~ms}$ would encounter the compression point, set $\sim 20 \mathrm{~dB}$ above the level to which the AGC regulates the 

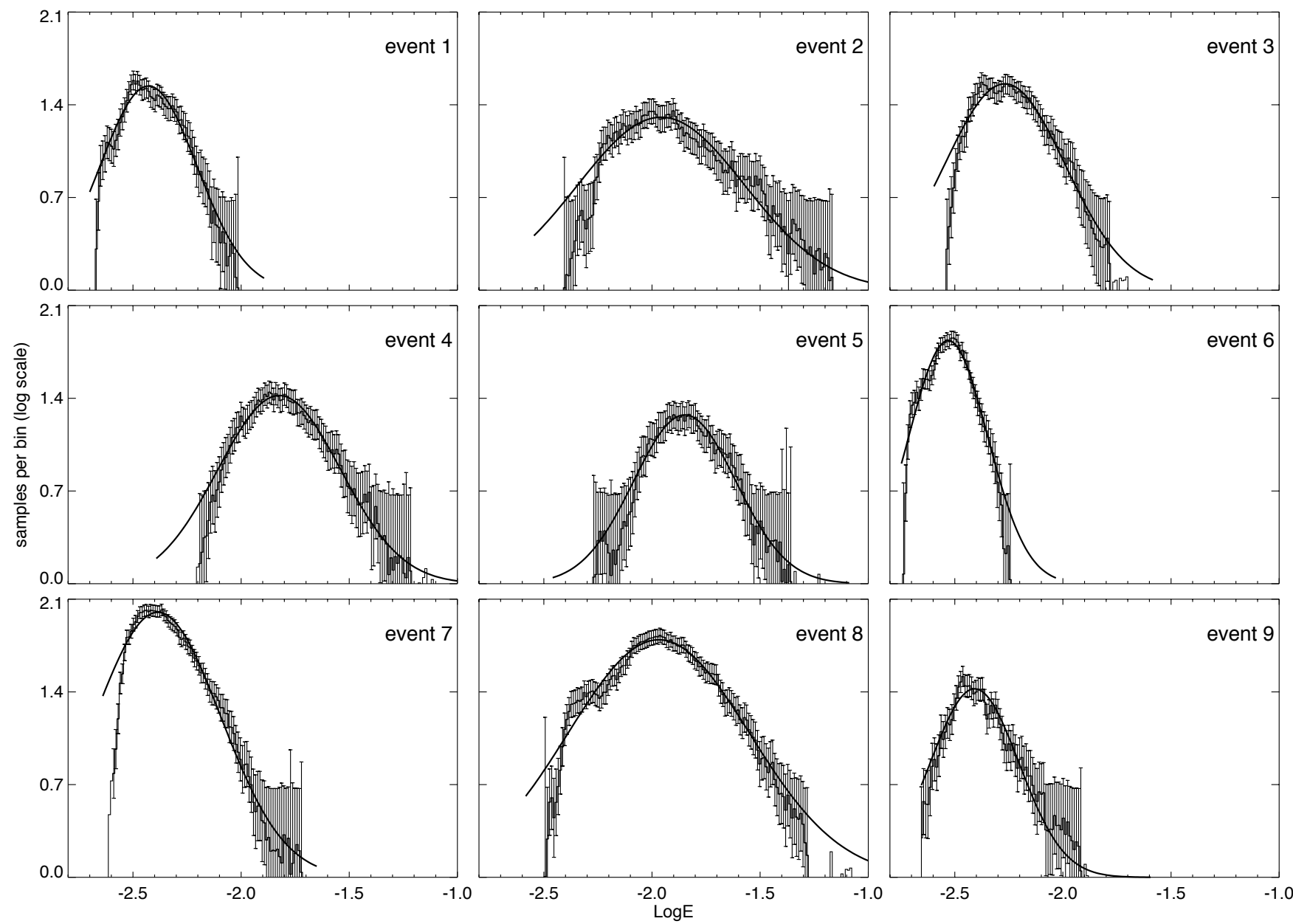

Fig. 5. Histograms of the effective number of samples, $N_{\text {eff }}$, versus the logarithm of the electric field amplitude, applying a $2.6 \mathrm{~ms}$ running average, for all nine events labeled in Fig. 1b. Lighter traces show Gaussian models (in $\log E$ )) fitted to the observed range of fields. Error bars reflect estimates of statistical uncertainty as described in the text.

signals. Signals increasing by more than about $20 \mathrm{~dB}$ in $1.6 \mathrm{~ms}$ would experience compression and would be underestimated in the recovered waveform. Similarly, a large enough decrease within $1.6 \mathrm{~ms}$ would cause the Langmuir wave signal to noise level to become small, in which case the signal would be overestimated in the recovered waveform. A decrease of order $10-15 \mathrm{~dB}$ within $1.6 \mathrm{~ms}$ would cause the signal level to approach the telemetry link noise level, assuming that the signal level is indeed dominated by the Langmuir wave and not set by some other signal.

We can evaluate these AGC-related instrumental effects by examining the raw HF data before they are corrected for AGC action. First, we confirmed that the Langmuir wave at $350-400 \mathrm{kHz}$ indeed dominates the raw waveform in the selected events. Second, we produced histograms of the raw signals. Figure 6 shows such a histogram of the positive side of the envelope of the Langmuir waves for event 1 , corresponding to $463.55-466.4 \mathrm{~s}$. The $\mathrm{x}$-axis is the raw signal level (in integer counts, $0-255$, where 128 counts corre- sponds to zero voltage), and the y-axis displays number of samples. Figure 6 includes only one sample per Langmuir wave period, that corresponds to the largest voltage reached in each cycle, and hence contains only a small fraction of the total raw data samples. Significantly, the raw amplitudes in Fig. 6 never approach the compression point, which is near 255 counts. Furthermore, only 505 out of 100000 samples, or $0.5 \%$, have values $\leq 130$ counts, which is $10 \mathrm{~dB}$ below the peak of 136 counts. Under these conditions, we expect no significant distortion due to AGC action. Even if the AGC time constant matched the transition time of $0.3 \mathrm{~ms}$ observed in the Langmuir waves statistics, there is no known mechanism by which the AGC would produce the observed transition or affect the distributions of electric fields calculated with a $2.6 \mathrm{~ms}$ running average.

If the transition is not instrumental, one possible interpretation is that for running averages shorter than $\sim 0.3 \mathrm{~ms}$ the statistics reflect the variation of the fields within one packet of Langmuir waves, whereas for running averages 


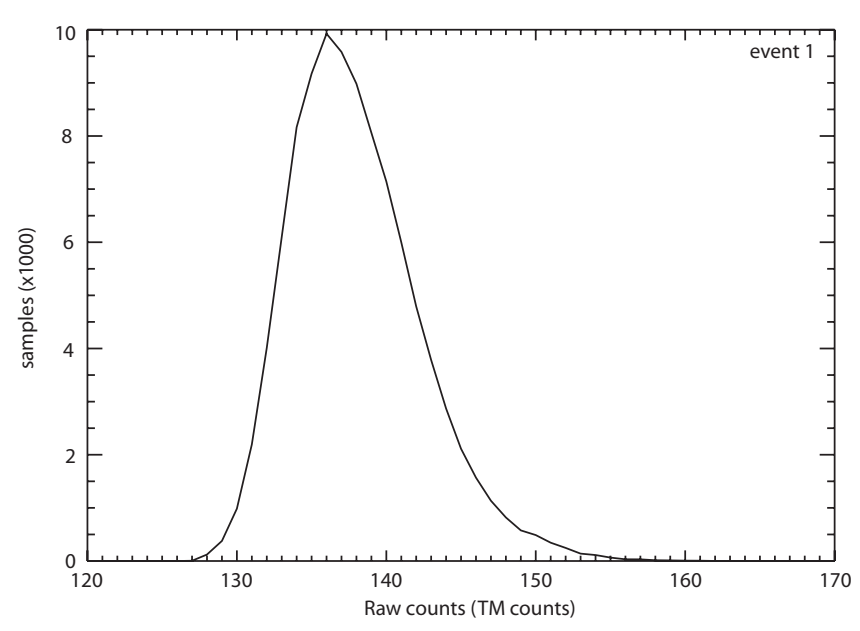

Fig. 6. Histogram of absolute value of the raw HF data, sampled twice per Langmuir wave period, at the peak values. The $\mathrm{x}$-axis is the raw signal level in integer counts (0-255) and the y-axis displays the number of samples.

much longer than $\sim 0.3 \mathrm{~ms}$, the distribution begins to be significantly affected by the statistics of wave packets; i.e., if the characteristic timescale for Langmuir wavepackets is a few $\mathrm{ms}$ then averaging over smaller times will lead to at most one wavepacket within the sample and a distribution dominated by the thermal noise expected between wavepackets, while averaging over longer time periods will correspond to averages over several wavepackets and little contribution from thermal noise.

A related possibility is that the transition time scale reflects whether the measurements correspond to temporallygrowing significantly nonthermal signals or to thermal plasma noise (Meyer-Vernet and Perche, 1989). Equation (4) of Cairns et al. (2000) shows that the characteristic thermal noise electric field is of order $0.3 \mathrm{mV} / \mathrm{m}$ for a $2000 \mathrm{~K}$ plasma with plasma frequency of $400 \mathrm{kHz}$. In comparison, the peaks in the observed probability distributions are near a few $\mathrm{mV} / \mathrm{m}$ (Figs. 2-4) and so are about a factor of 10 above the calculated thermal level. Therefore the transition, which affects the bulk of the sampled electric field levels, cannot be attributed to a transition from thermal to nonthermal signals. In principle, thermal noise can make a contribution to these types of observations, but the instrument noise level, $\sim 3 \mathrm{mV} / \mathrm{m}$ rms over the $5 \mathrm{MHz}$ bandwidth, implies that thermal waves make a negligible contribution to the measured field distributions.

We turn now to the shapes of the observed distributions of electric field amplitudes, which, to within the estimated uncertainties, are very well fitted by a lognormal form over the entire range of observed fields, above the instrument noise level, for all nine selected Langmuir bursts. This result establishes that the bursty waves are consistent with being in a stochastic growth state and well described by pure SGT.
SGT involves waves driven by a linear instability and subject to damping and scattering that vary with position and time in an inhomogeneous medium (Robinson, 1992, 1995; Robinson et al., 1993; Robinson and Cairns, 2001; Cairns and Menietti, 2001). Wave-particle interactions then lead to the system evolving to a state near marginal stability in which the wave gain $G=\int d t \Gamma=\ln \left(E / E_{0}\right)$ is a stochastic random variable. The waves are then intrinsically bursty and the free energy source can then persist longer than otherwise expected. Accordingly, SGT predicts a lognormal distribution of wave fields (and energy), provided only that the Central Limit Theorem applies. A semi-quantitative analytic model for the feedback mechanism exists for Langmuir waves interacting with an electron beam in an inhomogenous plasma (Robinson et al., 1993; Cairns and Robinson, 1997; Cairns and Menietti, 2001) and recent simulations show evolution of electron beam-Langmuir wave systems to a SGT state ( $\mathrm{Li}$ et al., 2006).

SGT was originally applied to Langmuir waves driven by electron beams in interplanetary type III radio sources (Robinson, 1993; Robinson et al., 1993). It has since been applied to similar beam-driven Langmuir waves in the foreshocks of Earth and other planets (Cairns and Robinson, 1997, 1999; Boshuizen et al., 2001; Sigsbee et al., 2004; Krasnoselskikh et al., 2007) and type II bursts (Knock et al., 2001), mirror mode and electromagnetic ion cyclotron waves in Earth's magnetosheath (Cairns and Grubits, 2001), and waves near the electron plasma frequency in Earth's inner magnetosphere and polar cap (Burinskaya et al., 2000; Cairns and Menietti, 2001). These regions are all much more weakly magnetized $\left(f_{c e} / f_{p e} \lesssim 0.1\right)$ than the auroral context considered in this paper $\left(f_{p e} \gtrsim f_{c e}\right)$. It might then be questioned whether SGT is likely to apply. However, many components of the extremely intense, coherent, radio emissions from pulsars have statistics consistent with SGT (Cairns, 2004), and these sources are believed to be extremely highly magnetized with $f_{c e} \gg f_{p e}$. In addition, the basic theory makes no assumptions on the relative sizes of $f_{c e}$ and $f_{p e}$.

As discussed above, the Langmuir waves analyzed in this paper are most plausibly driven by auroral electron beams. The situation then appears qualitatively analogous to the electron beam-driven Langmuir waves in type III sources and planetary foreshocks and over Earth's polar cap. It is then not surprising that the observed statistics are quantitatively consistent with SGT. Two scenarios by which the auroral waves and electron beams might evolve to an SGT state are as follows:

1. Spatiotemporal inhomogeneities in the background plasma lead to different wave growth in different regions, resulting in temporal and spatial fluctuations in the driving beam.

2. The auroral electron acceleration processes may be intrinsically bursty and unsteady, so that even initially the beam consists of beamlets with varying duration, speed, 
density, and temperature. This second scenario may be viewed as a way to "jumpstart" SGT, rather than relying upon the first scenario's wave-electron-medium interactions to produce the SGT state.

A quantitative means to test the first scenario exists for electron beam-driven Langmuir waves in an essentially unmagnetized enviroment (Robinson et al., 1993; Cairns and Robinson, 1997). It predicts that SGT should apply in type III sources and Earth's foreshock, as observed. Moreover, naive application of this method to the conditions of the PHAZEII experiment, which is a strongly magnetized environment, suggest that SGT should apply there (see Samara, 2005). However, detailed consideration of the mechanism by which the auroral electron beams and Langmuir waves evolve to an SGT state is deferred to further work.

The finding that the probability distributions of wave fields associated with nine Langmuir bursts observed in the PHAZE-II rocket experiment are quantitatively consistent with pure SGT suggests that other processes play small roles in the events studied. For instance, there is no evidence that the thermal distribution of waves is observed at low fields (Robinson, 1995; Cairns et al., 2000), even though the calculated total field of thermal Langmuir waves is $\approx 0.3 \mathrm{mV} / \mathrm{m}$, but this may be due to the instrumental noise level being higher $(\approx 3 \mathrm{mV} / \mathrm{m}$ integrated over the $5 \mathrm{MHz}$ bandwidth of the experiment). Likewise, there is no evidence for the characteristic high electric field cutoffs caused by nonlinear decay processes of the Langmuir waves (Robinson et al., 1993; Cairns et al., 2000). Modulational instabilities and strong turbulence wave collapse might also alter the field statistics at high $E$, likely causing power-laws (Robinson, 1997; Cairns, 2004). Despite evidence for such processes in other auroral Langmuir wave data sets (Ergun et al., 1991b; Khotyaintsev et al., 2001), the distributions of Langmuir wave electric fields analyzed here show no evidence for such power-law deviations from pure SGT. Nevertheless, in the Appendix we consider the constraints on collapsing wave packets and find that collapse cannot be ruled out. It therefore may be warranted to examine the collapse hypothesis in more detail, for example by applying magnetized theories to the auroral case to predict effects on the wave statistics, or by seeking examples of localized wavepackets in auroral rocket data and comparing them with the predictions of collapse theory.

\section{Conclusions}

Statistics of Langmuir wave electric field amplitudes observed with the HFE wave receiver on the PHAZE-II rocket in the auroral zone at altitudes 830 to $945 \mathrm{~km}$ reveal two noteworthy features. First, the distribution of running averages of the electric field amplitude takes on a similar form when the width of running averages is in the range $1-26 \mu \mathrm{s}$, then takes on a different form when the width is in the range 1-26 ms.
The transition time scale lies near $0.3 \mathrm{~ms}$. Second, for 2.6ms running averages, corresponding to widths exceeding this transition time scale, the distribution of the logarithm of electric field amplitudes matches a lognormal form to within uncertainty across the entire range of observed fields in all nine cases studied in detail. The field statistics are thus consistent with pure SGT. This suggests that the waves are driven by electron beams into an SGT state in the inhomogeneous auroral ionosphere, with the beam-wave-plasma system being close to a state of marginal stability. This is analogous to numerous other wave modes in other contexts. Other processes appear to play at most small roles in these events. However, wave collapse cannot be ruled out at this time for the shortest scale wavepackets and largest fields observed. Observational searches for such wavepackets and more exact theoretical calculations should be performed. Upcoming rocket launches should test these analyses and results, by providing more data with improved instrumentation that will allow better assessment of the approach to an SGT state and the robustness of the finding that SGT is relevant, further investigation and interpretation of the observed transition time scale, and evidence for other processes.

\section{Appendix A}

\section{Conditions for wave collapse in the auroral ionosphere}

Wave collapse is an alternative mechanism to SGT for forming bursty, spiky, Langmuir waves. It is a nonlinear self-focusing (or modulational) process (Zakharov, 1972; Nicholson et al., 1978; Robinson, 1997), in which wave energy refracts into the density depression associated with a wavepacket, intensifies the field and the nonlinear ponderomotive force, which then further evacuates the plasma and causes the wavepacket's scale size to decrease, leading to more refraction and further collapse of the scale size. A wavepacket of a given spatial scale $L$ has a threshold electric field for collapse to occur. This threshold electric field has not been calculated for the magnetized case. However, where comparisons have been done, the unmagnetized and magnetized treatments have given nearly identical qualitative results and similar quantitative results (Robinson, 1997). For example, in the magnetized case the wave packet shape changes slightly from the isotropic/spherical shape in the nonmagnetized case, and the power law exponent of the electric field statistics differs by $\sim 1$.

Because there is no analytical magnetized threshold to refer to, we consider here the non-magnetized case. Cairns and Robinson (1995) show that in this case a collapsing wave packet satisfies

$E_{a}(L)>\sqrt{\frac{170 n k_{B} T_{e}}{\epsilon_{0}}} \frac{\lambda_{D}}{L}$, 
where $L$ is the packet scale size and $E_{a}(L)$ is the envelope electric field. For the PHAZE II experiment we assume $n=2200 \mathrm{~cm}^{-3}$ and $T_{e}=3000 \mathrm{~K}$. Taking $L \simeq 0.5 \mathrm{~km}$, corresponding to the smallest possible perpendicular wavelength consistent with $k_{\|} / k_{\perp} \gg 1$ as discussed in the Discussion section, we obtain $E_{a}(L) \geq 6.74 \mathrm{mV} / \mathrm{m}$. This estimate represents an upper bound for the threshold field since assuming a larger perpendicular scale reduces the inferred threshold field.

In the presence of background density irregularities collapse may be disrupted, effectively requiring a larger field $E_{a}(L)$ for collapse to proceed. In that case there is an additional constraint imposed by the requirement that the packet collapses before density fluctuations disrupt it (Cairns and Robinson, 1995):

$E_{a}(L) \geq \sqrt{\frac{170 n k_{B} T_{e} V_{\mathrm{S}}}{\epsilon_{0} V_{e}}} \sqrt{\frac{\lambda_{D}}{L}}$.

Using $m_{i}=16 m_{p}$ (for oxygen) and assuming that $T_{e} \approx T_{i}$ we obtain $E_{a}(L) \geq 41 \mathrm{mV} / \mathrm{m}$. The PHAZE II electric fields range from 3 to $158 \mathrm{mV} / \mathrm{m}$, thereby exceeding the first constraint but within the range of the second one for the specific range of plausible wavepacket lengthscales used. These rough calculations, based on unmagnetized theory, suggest that collapse cannot be excluded. It therefore may be warranted to examine the collapse hypothesis more exactly, by applying magnetized theories to the auroral case and also by searching auroral rocket data for localized wavepackets and then comparing them with collapse theory.

Acknowledgements. The work at Dartmouth College was supported by NASA grants NNG04WC27G, NNG05WC44G, NNG05GM37G, and NNG07AK03G. The work at the University of Sydney was supported by the Australian Research Council. Special thanks to Southwest Research Institute. The authors thank Professor Rudolf Treumann for extensive discussions.

Topical Editor M. Pinnock thanks J. D. Menietti and another anonymous referee for their help in evaluating this paper.

\section{References}

Arnoldy, R. L., Lynch, K. A., Austin, J. B., and Kintner, P. M.: Energy and pitch-angle dispersed auroral electrons suggesting a time-variable, inverted-V potential structure, J. Geophys. Res., 104(A10), 22 613-22 622, 1999.

Beghin, C., Rauch, J. L., and Bosqued, J. M.: Electrostatic plasma waves and HF auroral hiss genarated at low altitude, J. Geophys. Res., 94, 1359-1378, 1989.

Boehm, M.: Waves and static electric fields in the auroral acceleration region, $\mathrm{PhD}$ thesis, University of California, Berkeley, 1987.

Bonnell, J., Kintner, P., Wahlund, J.-E., and Holtet, J. A.: Modulated Langmuir waves: Observations from Freja and SCIFER, J. Geophys. Res., 102, 17 233-17 240, 1997.

Boshuizen, C. R., Cairns, I. H., and Robinson, P. A.: Stochastic growth theory of spatially-averaged distributions of Langmuir fields in Earth's foreshock, Geophys. Res. Lett., 28, 3569-3572, 2001.
Burinskaya, T. M., Rusanov, A. A., and Mogilevsky, M. M.: Smallscale bursts of the Langmuir oscillations in the polar cap, Cosmic Res., 38, 475-482, 2000.

Cairns, I. H.: Properties and interpretations of giant micropulses and giant pulses from pulsars, Astrophys. J., 610, 948-955, 2004.

Cairns, I. H. and Grubits, K. A.: Stochastic growth of ion cyclotron and mirror waves in Earth's magnetosheath, Phys. Rev., 64, 56 408-56411, 2001.

Cairns, I. H. and Melrose, D. B.: A theory for the $2 f_{p}$ radiation upstream of the Earth's bow shock, J. Geophys. Res., 90, 66376640, 1985.

Cairns, I. H. and Menietti, J. D.: Stochastic growth of waves over Earth's polar cap, J. Geophys. Res., 106, 29 515-29 530, 2001.

Cairns, I. H. and Robinson, P. A.: Strong Langmuir turbulence at Jupiter?, Geophys. Res. Lett., 19, 1069-1072, 1992.

Cairns, I. H. and Robinson, P. A.: Inconsistency of Ulysses millisecond Langmuir spikes with wave collapse in type III radio sources, Geophys. Res. Lett., 22, 3437-3440, 1995.

Cairns, I. H. and Robinson, P. A.: First test of stochastic growth theory for Langmuir waves in Earth's foreshock, Geophys. Res. Lett., 24, 369-372, 1997.

Cairns, I. H. and Robinson, P. A.: Strong evidence for stochastic growth of Langmuir-like waves in Earth's foreshock, Phys. Rev. Lett., 82, 3066-3069, 1999.

Cairns, I. H., Robinson, P. A., and Anderson, R. R.: Thermal and driven stochastic growth of Langmuir waves in the solar wind and Earth's foreshock, Geophys. Res. Lett., 27, 61-64, 2000.

Cairns, I. H., Robinson, P. A., and Smith, N. I.: Arguments against modulational instability of Langmuir waves in Earth's foreshock, J. Geophys. Res., 103, 287-300, 1998.

Ergun, R. E., Carlson, C. W., MacFadden, J. P., Clemmons, J. H., and Boehm, M. H.: Langmuir wave growth and electron bunching: Results from a wave-particle correlator, J. Geophys. Res., 96, 225-238, 1991a.

Ergun, R. E., Carlson, C. W., MacFadden, J. P., Clemmons, J. H., and Boehm, M. H.: Evidence of a transverse Langmuir modulational instability in a space plasma, Geophys. Res. Lett., 18, 1177-1180, 1991b.

Ergun, R. E., Carlson, C. W., McFadden, J. P., Mozer, F. S., Delory, G. T., Peria, W., Chaston, C. C., Temerin, M., Roth, I., Muschietti, L., Elphic, R., Strangeway, R., Pfaff, R., Cattell, C. A., Klumpar, D., Shelley, E., Peterson, W., Moebius, E., and Kistler, L.: FAST satellite observations of large-amplitude solitary structures, Geophys. Res. Lett., 25, 2041-2044, 1998.

Goldman, M. V., Oppenheim, M. M., and Newman, D. L.: Nonlinear two-stream instabilities as an explanation for auroral bipolar wave structures, Geophys. Res. Lett., 26, 1821-1824, 1999.

Khotyaintsev, Yu., Lizunov, G., and Stasiewicz, K.: Langmuir wave structures registered by FREJA: analysis and modeling Adv. Space Res., 28, 1649-1654, 2001.

Knock, S. A., Cairns, I. H., Robinson, P. A., and Kuncic, Z.: Theory of type II radio emission from the foreshock of an interplanetary shock, J. Geophys. Res., 106, 25 041-25 052, 2001.

Krasnoselskikh, V. V., Lobzin, V. V., Musatenko, K., Soucek, J., Pickett, J. S., and Cairns, I. H.: Beam-plasma interaction in randomly inhomogeneous plasmas and statistical properties of small-amplitude Langmuir waves in the solar wind and electron foreshock, J. Geophys. Res., 112, A10109, doi:10.1029/2006JA012212, 2007. 
LaBelle, J., McAdams, K. L., and Trimpi, M. L.: High-frequency and time resolution rocket observations of structured low- and medium-frequency whistler mode emissions in the auroral ionosphere, J. Geophys. Res., 104, 28 101-28 107, 1999.

Li, B., Robinson, P. A., and Cairns, I. H.: Numerical simulations of type III solar radio bursts, Phys. Rev. Lett., 96(14), 145005 , doi:10.1103PhysRevLett.96.145005, 2006.

Lin, R. P., Levedahl, W. K., Lotko, W., Gurnett, D. A., and Scarf, F. L.: Evidence for nonlinear wave-wave interactions in solar type III radio bursts, ApJ., 308, 954-965, 1986.

Lizunov, G. Y., Khotyaintsev, Y., and Stasiewicz, K.: Parametric decay to lower hybrid waves as a source of modulated Langmuir waves in the topside ionosphere, J. Geophys. Res., 106, 24755 $24763,2001$.

Lynch, K. A., Arnoldy, R. L., Kintner, P. M., Schuck, P., Bonnell, J. W., and Coffey, V.: Auroral ion acceleration from lower hybrid solitary structures: A summary of sounding rocket observations, J. Geophys. Res., 104, 28 515-28 534, 1999.

McAdams, K. L., LaBelle, J., Schuck, P. W., and Kintner, P. M.: PHAZE II observations of lower hybrid burst structures occuring on density gradients, Geophys. Res. Lett., 25, 3091-3094, 1998.

McAdams, K. L. and LaBelle, J.: Narrowband structrure in HF waves above the electron plasma frequency in the auroral ionosphere, Geophys. Res. Lett., 26, 1825-1828, 1999.

McAdams, K. L., LaBelle, J., Trimpi, M. L., Kintner, P. M., and Arnoldy, R. A.: Rocket observations of banded structure in waves near the Langmuir frequency in the auroral ionosphere, J. Geophys. Res., 104, 28 109-28 122, 1999.

McAdams, K. L., Ergun, R. E., and LaBelle, J.: HF chirps: Eigenmode trapping in density depletions, Geophys. Res. Lett., 27, 321-324, 2000.

Meyer-Vernet, N. and Perche, C.: Tool kit for antennae and thermal noise near the plasma frequency, J. Geophys. Res., 94, 2405$2415,1989$.

Muschietti, L., Roth, I., and Ergun, R. E.: Kinetic localization of beam-driven Langmuir waves, J. Geophys. Res., 100, $17481-$ $17490,1995$.

Muschietti, L., Roth, I., and Ergun, R. E.: On the formation of wave packets in planetary foreshocks, J. Geophys. Res., 101, 15 605$15613,1996$.

Muschietti, L., Ergun, R. E., Roth, I., and Carlson, C. W.: Phasespace electron holes along magnetic field lines, Geophys. Res. Lett., 26, 1093-1096, 1999a.

Muschietti, L., Roth, I., Ergun, R. E., and Carlson, C. W.: Analysis and simulation of BGK electron holes, Nonlin. Processes Geophys., 6, 211-219, 1999b, http://www.nonlin-processes-geophys.net/6/211/1999/.

Muschietti, L., Roth, I., Carlson, C. W., and Ergun, R. E.: Transverse instability of magnetized electron holes, Phys. Rev. Lett., 85, 94-97, 2000.
Muschietti, L., Roth, I., Carlson, C. W., and Berthomier, M.: Modeling stretched solitary waves along magnetic field lines, Nonlin. Processes Geophys., 9, 101-109, 2002, http://www.nonlin-processes-geophys.net/9/101/2002/.

Newman, D. L., Goldman, M. V., Ergun, R. E., and Boehm, M. H.: Langmuir turbulence in the auroral ionosphere 1: Linear theory, J. Geophys. Res., 99, 6367-6376, 1994a.

Newman, D. L., Goldman, M. V., and Ergun, R. E.: Langmuir turbulence in the auroral ionosphere 2 : Nonlinear theory and simulations, J. Geophys. Res., 99, 6377-6391, 1994b.

Newman, D. L., Goldman, M. V., Spector, M., and Perez, F.: Dynamics and instability of electron phase-space tubes, Phys. Rev. Lett., 86, 1239-1242, 2001.

Nicholson, D. R., Goldman, M. V., Hoyng, P., and Weatherall, J. C.: Nonlinear Langmuir waves during type III solar radio bursts, ApJ., 223, 605-619, 1978.

Oppenheim, M. M., Newman, D. L., and Goldman, M. V.: Evolution of electron phase-space holes in a 2D magnetized plasma, Phys. Rev. Lett., 83, 2344-2347, 1999.

Oppenheim, M. M., Vetoulis, G., Newman, D. L., and Goldman, M. V.: Evolution of electron phase-space holes in 3D, Geophys. Res. Lett., 28, 1891-1894, 2001.

Robinson, P. A.: Clumpy Langmuir waves in type III radio sources, Solar Phys., 139, 147-163, 1992.

Robinson, P. A.: Stochastic-growth theory of Langmuir growth-rate fluctuations in type III radio sources, Solar Phys., 146, 357-363, 1993.

Robinson, P. A., Cairns, I. H., and Gurnett, D. A.: Clumpy Langmuir waves in type III solar radio sources: Comparison of stochastic-growth theory with observations, ApJ., 407, 790-800, 1993.

Robinson, P. A.: Stochastic wave growth, Phys. Plasmas, 2, 14661479, 1995.

Robinson, P. A.: Nonlinear wave collapse and strong turbulence, Rev. Mod. Phys., 69, 507-573, 1997.

Robinson, P. A. and Cairns, I. H.: Stochastic growth of localized plasma waves, Phys. Plasmas, 8, 2394-2400, 2001.

Samara, M.: Sounding Rocket Investigations of Whistler, UpperHybrid, and Langmuir Waves in the Auroral Ionosphere, $\mathrm{PhD}$ thesis, Dartmouth College, Hanover, New Hampshire, 2005.

Sigsbee, K., Kletzing, C., Gurnett, D. A., Pickett, J. S., Baloogh, A., and Lucek, E.: The dependence of Langmuir wave amplitudes on position in Earth's foreshock, Geophys. Res. Lett., 31, L07805, doi:10.1029/2004GL019413, 2004.

Zakharov, V. E.: Collapse of Langmuir waves, Sov. Phys. JETP, 35, 908-914, 1972. 